\title{
On Linear Algebra for Non Mathematics Majors
}

\author{
Huaichen Chen ${ }^{*}$, Shuping Gao², Wei Yang3 \\ ${ }^{1}$ Department of Electronic Engineering, Xidian University, Xi'an, China \\ ${ }^{2}$ Department of Science, Xidian University, Xi'an, China \\ ${ }^{3}$ Department of Technical Physics, Xidian University, Xi'an, China \\ Email: "hchchen1934@vip.163.com,gaosp@mail.xidian.edu.cn, weiyang@mail.xidian.edu.cn
}

Received 14 January 2016; accepted 6 April 2016; published 13 April 2016

\begin{abstract}
To find out what knowledge in linear algebra is essential to non-mathematics students, a reverse tracking method was used. Based on practical problems likely to encountered by students in subsequent engineering courses, the minimum contents required has been determined. Rules are proposed to meet the background of most freshman students. An application oriented, easy to understand, computer based text book "Applied Popular Linear Algebra with MATLAB" [1] was published.
\end{abstract}

\section{Keywords}

Linear Algebra, Determinant, Matrix Operation, Vector Space, Visualizing Teaching, Gaussian Elimination, Rank, Singular Value Decomposition, qr Decomposition, Computerized Solution, MATLAB

\section{Why Linear Algebra Is So Important in Modern Engineering Universities?}

Linear algebra originated in 18th Century. Until 1950's, it was only studied by mathematics students and some graduate students of other areas. But after 1960, it was included in the undergraduate curriculum of the non Mathematics Departments of American Universities. In China, the engineering departments offered no linear algebra until 1980s. Why is linear algebra so popular in recent decades? The most fundamental reason is that it is the basis of many algorithms for the machine implementation of numerous technical problems. In Prof. Leontiff's work leading to the 1973 economics Nobel Prize, he used computer to solve the 42-th order of linear equation group. In early 80’s, linear algebra software package LINPACK was developed. So users can solve the problem of large complex linear algebra without mastering the mathematical details of matrix theory. LINPACK is also the background support of MATLAB. The achievements of linear algebra in the last half of the 20th century also focused on solving the problems of large-scale matrix using computer. It is no longer a pure theory of mathematics but becomes a calculation tool for common engineering students.

Modern society has put forward higher requirements for engineering mathematics. In 1989, Dr. Tsien Hsueshen wrote: "In the future, solving a problem can all rely on the computer. People do not need to calculate one

${ }^{*}$ Corresponding author. 
point after another. But until today, the mathematics of engineering is built on the calculation by people themselves, ... The mathematics course of science and engineering must be reformed. It should not focus on solving problems by students themselves, but let them learn to command machine in solving problems and to understand the answers given by the computer...” [2]. Linear algebra is the basis of numerical calculation, which not only takes the lead in the use of computer, but also promotes the computer applications based on other mathematic courses and engineering problems.

The importance of linear algebra is also reflected in the increasing number of new fields using multivariable matrix models. Leontiff's story was a good example. This kind of "demand pull” for non-mathematics major is in the use of computer to solve high-order complex matrix model, not in the manual deduction of mathematical formula. Its contents are very different to the classical linear algebra. At the beginning, no one had a clear idea, the curriculum of mathematics department was copied to teach the non-mathematics students. But the task for non mathematical students is to solve real world problems. What they face is high order, numerical complex matrices models. Their different requirement can be summed up in Table 1.

After implementation for decades, some famous professors in the USA became seriously concerned. They set up a Linear Algebra Curriculum Study Group (LACSG) in 1990. Five recommendations of linear algebra as the first course were put forward [3]:

1) It is to meet the needs of non-mathematics students;

2) It should be oriented to matrix operation instead of vector space;

3) It should be adapted to the level and needs of students (freshman or sophmore);

4) It should use the latest computing technology;

5) Abstract contents should be omitted and left to subsequent courses.

These five recommendations clearly point out the major differences between the new and original linear algebra curriculum. The first four address mainly the requirements of linear algebra for high-order real world systems. The fifth postpones the abstract contents to subsequent courses. To provide instructional staffs for the new curriculum, the NSF-sponsored an ATLAST program. In six years, they trained a large number of professors in using MATLAB to solve linear algebra problems.

Since 2009, a project: "Using MATLAB and Modeling to Reform Engineering Linear Algebra Course" was set up by the Ministry of Higher Education in China. It was proposed and hosted by Xidian University. 19 universities participated in and implemented for two years. It involved 200 professors and 45,000 students. Now it is a consensus among these universities to require their engineering freshmen to learn MATLAB and the use of computers. This laid the first stone of computer usage in the undergraduate curriculum.

Under the constraint of credit hour to the course of linear algebra, there is no sufficient time to cover adequately both the classical contents of linear algebra and its MATLAB programming. Thus it is of practical importance to determine the minimum part of linear algebra essential to engineering implementation.

\section{How to Meet the Needs of Non Mathematics Majors?}

To realize this, one must go deep into the practice of non-mathematics majors. We found that matrix equation is the core. The author had programmed more than 200 application in a dozen or more subsequent courses, this book took 46 cases among them. These including the following: (1) Matrix calculation and inversion in 12 cases (highest order 21); (2) Solving linear equations 16 cases (highest order 7); (3) Vector spatial relationship and motion transform 8 cases, (involving spatial geometry and motion, coplanarity problems); (4) linear transformation and characteristic roots 10 cases (containing complex eigenvalue problems). Other than some higher-order problems, the 46 cases in this book cover practically most application types in undergraduate courses.

Table 1. Different features of linear algebra for math and non-math major.

\begin{tabular}{ccc}
\hline & Mathematics Department & Non mathematics Majors \\
\hline Matrix order & Less than 4 & Any high order (up to hundreds) \\
Theory & Classical theory for small matrices & Theory adapt to large matrices \\
Tools and results & Pencil, symbolic formula & Computer, numerical \& symbolic results \\
Emphasized on & Abstract thinking of N-dimension & 3-D spatial concept \\
Applications & Few examples of small integer matrices & Real world complex problems in many area \\
\hline
\end{tabular}


Method of reverse thinking is used to extract from these examples the minimum required linear algebra theory. All theories needed by these cases were kept and explain in detail. Any contents not directly used are removed. Some complicated theory is simplified if possible. After all, engineering talent is the use of mathematics, not engaged in mathematics itself. This book takes full advantage of MATLAB resources to link up theory with the subroutines, so that students will understand what and why they are doing.

The main points are as follows:

(1) Gaussian elimination method for solving linear equations is the core theory of the book. Determinant is simplified and defined as the product of the pivot elements in the row echelon form [4]. Rank, vector correlation, ... are defined accordingly. Under this definition, subroutine for row echelon form is often used. It can replace almost all determinant calculations.

(2) In order to integrate with computer in large scale numerical computations, students must have the concepts of computational complexity and accuracy which are never seen in the previous textbooks. The computational feasibility knock out two classical definitions of determinant in engineering. Error dependent rank is the key for solving coplanarity of vector group, it cannot be discovered by manual computation with integer elements. These examples show that our new concerns prevail the old ones.

(3) At the freshman level, we emphasized the relationship between linear algebra and 3-D solid analytic geometry. It is not necessary to teach N-D vector space before they clearly grasp 3-D space. Besides we cannot find any application of N-D in the undergraduate engineering courses.

Thus the contents and organization of this book are very different from the classical linear algebra textbooks.

\section{The Reform of Determinant Definition}

For the computation of determinant, the definition and method of its realization must be paid attention to. In numerical calculation, the computational complexity is usually measured by the number of multiplications. Table 2 gives a comparison of three methods with different matrix order:

Comparing method 1 and 3, we see that only when $n=2$, 3 , method 1 is better. When $n \geq 4$, method 1 and 2 are unable to calculate manually. When $n=25$, the multiplication number of these two definitions are too large that it would take hundreds of years to calculate a single determinant using a top computer today. This phenomenon is called "Dimensionality Curse". Clearly definitions 1 and 2 are useless to high order systems and can be ignored in textbook. For definition 3, not only the amount of calculation is greatly reduced, the formula is also very easy to program. Concepts like number of inversion, cofactors and its expansion, adjoint matrix, ... that discussed in the classical theory can all be omitted. Avoiding these "stumbling block" can greatly reduce the difficulty for the non-mathematics students and save many hours of instruction.

We found that in all the application cases, none of them really need determinant (except area and volume calculation which is not linear algebra problem). Many theorems on determinants and its nature are useless. This is because Gauss elimination method has already used nonzero criteria for pivot elements, which equivalents to determinant criteria. This means that determinant has already been used in Gaussian elimination, its further calculation is redundant. Pivot elements are the natural extension of Gauss elimination, no new concept or formula is needed. By using this definition, it is also easy to prove a variety of useful properties of determinant.

Someone takes the classical definition of determinant as the basis of linear algebra. This is not true. After proved that it has the fatal defect for computing high order matrix, we should not let the engineering students waste time on it. The era of the classical determinant is over! One of the supporting evidence is the software tools. In MATLAB, you cannot find terms or subroutines based upon these classical concepts. This means that real world do not need these concepts. The second evidence is some well known textbooks [5] [7]. In [5], Lay made a brilliant comment: "In Cauchy's day, when life was simple and matrices were small, determinant played a major role in mathematics. Today determinant is of little numerical value in large scale matrix computations.”

Table 2. Multiplications needed for different determinant definitions.

\begin{tabular}{ccccccc}
\hline Order $\mathrm{n}$ & 2 & 3 & 4 & 5 & 10 & 25 \\
\hline 1. Explicit Formula $\mathrm{N} \approx(\mathrm{n}-1) \mathrm{n} !$ & 2 & 12 & 72 & 480 & $32,659,200$ & $3.72 \times 10^{26}$ \\
2. Method of Cofactors $\mathrm{N} \approx 2 \mathrm{n} !$ & 2 & 9 & 40 & 205 & $7,257,600$ & $3.10 \times 10^{25}$ \\
3. Product of Pivots $\mathrm{N} \approx \mathrm{n}^{3} / 3$ & 4 & 13 & 24 & 45 & 342 & 5233 \\
\hline
\end{tabular}




\section{Give a Thorough Exposition to 3-D Vector Space}

Helping freshman students to establish three-dimension concept is an important goal in the university curriculum. Experiences indicate that it is not so easy. It needs efforts from many courses including linear algebra. Many Chinese textbooks were modified from senior grade math textbooks. Their emphasis is on abstraction and $\mathrm{N}$-dimensional space, which is apparently beyond the level of freshman students. An engineering oriented linear algebra textbook should combine algebra with geometry, this can be done only by stereogram in three-dimensional space. Emphasis on N-dimension makes a book full of formula without graphics, thus very hard to understand.

Many engineering applications are related to 3-D vector space, for example, the shape and movement of solids, position transformation in robotics and coordinate measuring instrument, projection of 3-D animation on 2-D screen, 3-D printers, etc. Even in linear algebra itself, the least square solution for over determined system can be easily derived using 3-D space vector model. There are so many 3-D unsolved problems in real world, why hurry up to N-D? So far no applications of N-D vector space in undergraduate courses were found. We suppose that mastering 3-D concept is good enough for the freshman engineering students, N-D abstract content should be aimed at the graduate and high grade mathematics students.

\section{Weaken the Underdetermined, Strengthen the Overdetermined}

We found that underdetermined applications are rarely seen, because they are mostly due to defect in the problem proposition. Engineer should either reject it or introduce supplement conditions to make it determined. We cannot find any usage of underdetermined solution set in real world as taught by classical linear algebra. For example, balancing chemical equations remains underdetermined unless you add a condition that the solution must be minimum positive integer numbers. It would be funny that you give the solution set as a super line in a virtual space instead of coefficients of each substances.

The over determined problems come from the inevitable interference and measurement errors in practice. They occur in many engineering problems. Finding the least mean square solution using geometric method involves the concept of vector space. But most of the textbook in China do not contain it. So many engineering problems are unsolvable to them. Over emphasizing underdetermined system but ignoring over determined problem is the major weakness. This caused linear algebra go far away from engineering. We should take a balanced way.

\section{Reduce the Order of Characteristic Equation but Allow Complex Roots}

In the classical textbooks, the characteristic equations are discussed in N-th order, but no book tells how to find the characteristic roots of even 3rd order by hand calculation. This is apparently inconsistent. Moreover, we cannot find their physical meaning and geometric model in undergraduate courses. So we restrict characteristic equations and quadratic forms to 2nd order, but included complex eigenvalues which can help to understand vibration problems in engineering.

\section{Brief Introduction to Some Examples That Demand for Course Reform [1]}

The following examples can prove the helplessness and necessity of reforming for classical theory.

\section{Example 1. Circuit as Figure 1, find $\mathbf{I}_{\mathrm{a}}, \mathbf{I}_{\mathrm{b}}, \mathbf{I}_{\mathrm{c}}$.}

The matrix model is:

$$
\left[\begin{array}{ccc}
R_{1}+R_{2}+R_{3} & -R_{3} & 0 \\
-R_{3} & R_{3}+R_{4}+R_{5} & -R_{5} \\
0 & -R_{5} & R_{5}+R_{6}+R_{7}
\end{array}\right]\left[\begin{array}{c}
I_{\mathrm{a}} \\
I_{\mathrm{b}} \\
I_{\mathrm{c}}
\end{array}\right]=\left[\begin{array}{c}
1 \\
0 \\
0
\end{array}\right] u_{\mathrm{s}} \Rightarrow \mathbf{A I}=\mathbf{b} \Rightarrow \mathbf{I}=\operatorname{inv}(\mathbf{A})^{*} \mathbf{b}
$$

In real world circuits, the matrix order could be dozens. In AC circuit, the variables $\mathbf{I}$ and coefficients $\mathbf{R}$ are complex numbers. It is impossible to solve the problem without computer software, while determinant calculation is not needed. We can get the solution easily without knowing anything about its determinant.

Example 2. Signal Flow Graph as Figure 2, find transfer function $w_{4}=x_{4} / \mathbf{u}$.

The equations and matrix model are as follows: 


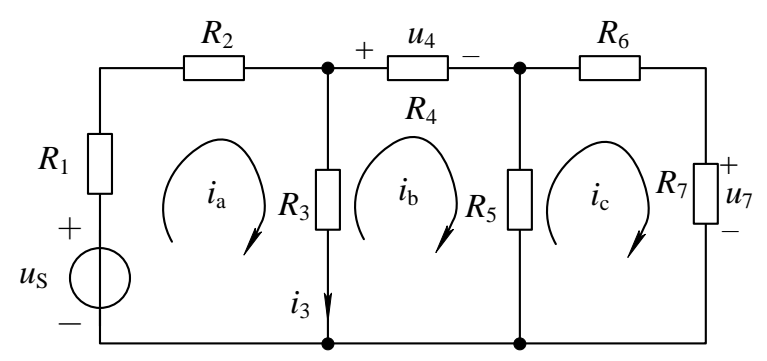

Figure 1. A resistance circuit.

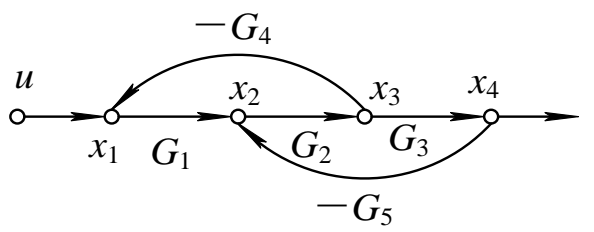

Figure 2. A signal flow graph.

$$
\left\{\begin{array}{l}
x_{1}=-G_{4} x_{3}+u \\
x_{2}=G_{1} x_{1}-G_{5} x_{4} \\
x_{3}=G_{2} x_{2} \\
x_{4}=G_{3} x_{3}
\end{array} \Rightarrow\left[\begin{array}{l}
x_{1} \\
x_{2} \\
x_{3} \\
x_{4}
\end{array}\right]=\left[\begin{array}{cccc}
0 & 0 & -G_{4} & 0 \\
G_{1} & 0 & 0 & -G_{5} \\
0 & G_{2} & 0 & 0 \\
0 & 0 & G_{3} & 0
\end{array}\right]\left[\begin{array}{l}
x_{1} \\
x_{2} \\
x_{3} \\
x_{4}
\end{array}\right]+\left[\begin{array}{l}
1 \\
0 \\
0 \\
0
\end{array}\right] u \Rightarrow \mathbf{X}=\mathbf{A X}+\mathbf{b u} \Rightarrow \frac{\mathbf{X}}{\mathbf{u}}=(\mathbf{I}-\mathbf{A})^{-\mathbf{1}} \mathbf{b}\right.
$$

The result given by MATLAB execution is:

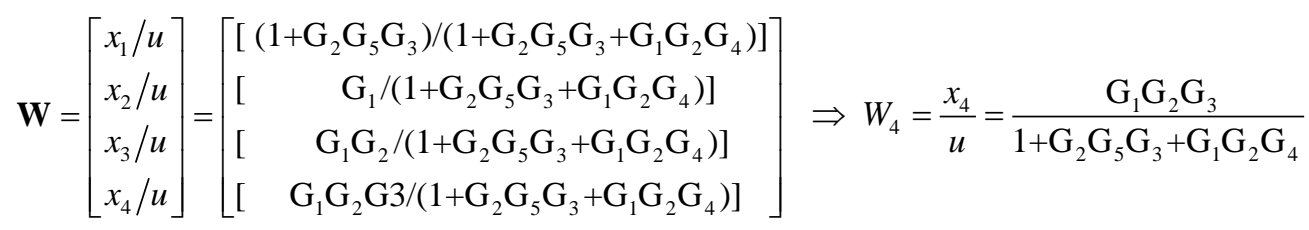

This method can also be used in digital filter (Figure 3) and control system (Figure 4) analysis to find out the overall system function. No matter how high its order is, using matrix operation supported software tools can give the solution in seconds.

The answer of Figure 3 is:

$$
W=\frac{y}{u}=-2 \frac{q+8}{(3 q+4)(q-2)}=\frac{-2 z^{-1}-16}{\left(3 z^{-1}+4\right)\left(z^{-1}-2\right)}
$$

The answer of Figure 4 is [6]:

$$
\mathbf{W}=\mathbf{X} / \mathbf{U}=\left[\begin{array}{cc}
x_{1} / u_{1} & x_{1} / u_{2} \\
x_{2} / u_{1} & x_{2} / u_{2} \\
x_{3} / u_{1} & x_{3} / u_{2} \\
x_{4} / u_{1} & x_{4} / u_{2} \\
e / u_{1} & e / u_{2}
\end{array}\right]=\frac{1}{1+G_{1} G_{2} G_{3}}\left[\begin{array}{ll}
1-G_{2} G_{3} G_{4} & -G_{3} \\
G_{1}+G_{4} & G_{1} G_{3} \\
G_{1} G_{2}+G_{2} G_{4} & -G_{1} G_{2} G_{3} \\
G_{1} G_{2}+G_{2} G_{4} & 1 \\
G_{1} G_{2}+G_{2} G_{4} & -G_{1} G_{2} G_{3}
\end{array}\right]
$$

This system has a $2 \times 1$ input vector and a $5 \times 1$ output vector, we obtain $5 \times 2$ transfer functions in one execution. This is a very important usage of linear algebra in subsequent courses. Using hand calculation could never get this privilege. These examples come from 3 subsequent important courses, so we can see the importance and power of linear algebra after reform to engineers.

Example 3. Evaluate coplanarity of 5 spatial points listed in Table 3.

This is a problem that can be encountered in modern mechanical workshop with coordinate measuring machine. The steps for solving this problem are as follows: 


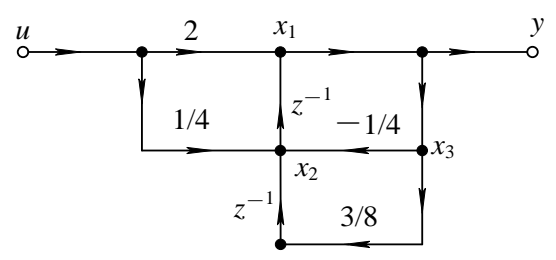

Figure 3. A digital filter.

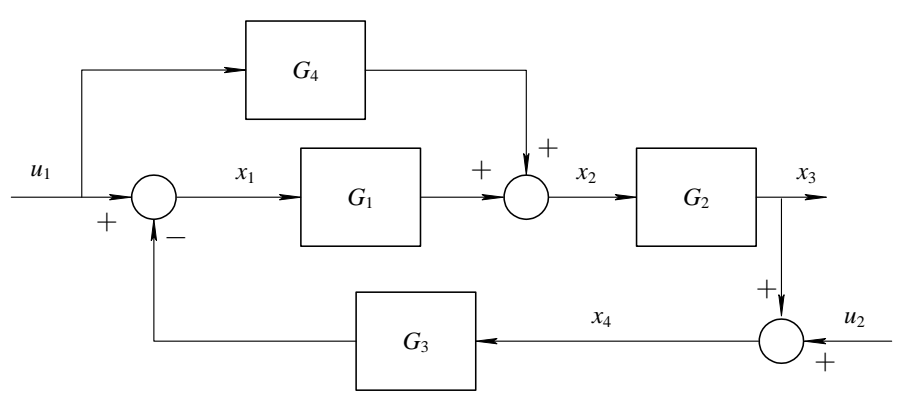

Figure 4. A control system.

Table 3. Coordinates measured for the 5 points.

\begin{tabular}{cccccc}
\hline & point a & point b & point c & point d & point $\mathrm{e}$ \\
\hline $\mathrm{x}$ & -0.28 & 4.00 & 0.72 & 2.70 & 2.00 \\
$\mathrm{y}$ & -0.03 & 4.60 & 0.71 & 0.20 & 1.80 \\
$\mathrm{z}$ & 0.55 & 3.00 & -0.13 & 6.20 & -0.32 \\
\hline
\end{tabular}

(1) Given vector group $V=\left[\mathrm{v}_{\mathrm{a}}, \mathrm{v}_{\mathrm{b}}, \mathrm{v}_{\mathrm{c}}, \mathrm{v}_{\mathrm{d}}, \mathrm{v}_{\mathrm{e}}\right]$, its columns are the coordinates of these points. Let $\mathrm{U}=\mathrm{V}-\left[\mathrm{v}_{\mathrm{e}}\right.$, $\mathrm{v}_{\mathrm{e}}, \mathrm{v}_{\mathrm{e}}, \mathrm{v}_{\mathrm{e}}, \mathrm{v}_{\mathrm{e}}$ ] be the difference vector group, which means the coordinate origin is moved to point $\mathrm{e}$.

(2) if the points are in a plane, the rank of $U$ must be 2 or less. Because of the limited data precision and computing error, precise plane can never realized. You will always find $\operatorname{rank}(\mathrm{U})=3$.

(3) The question is that in what extent these points are on the same plane. What is the biggest error? This relates to singular value decomposition which is too difficult. But it can be solved by software tools for non mathematic students. In MATLAB, function RANK (A, TOL) contains an tolerant argument TOL, its default value is $10^{-16}$. So very small distortion of the plane will cause $\operatorname{rank}(\mathrm{U})=3$. Using bigger TOL, we obtain rank $(\mathrm{U}, 0.01)$ $=3$ and $\operatorname{rank}(\mathrm{U}, 0.1)=2$. This means that these 5 points are about on a plane within an error of \pm 0.1 .

(4) To find the equation of the fitted plane and the distance of each point to it, the easiest way is to use qr decomposition. Inputting [Q,R] = qr(U), we get:

$$
\mathbf{Q}=\left[\begin{array}{ccc}
-0.75 & -0.08 & 0.66 \\
-0.60 & -0.35 & -0.72 \\
0.29 & -0.93 & 0.21
\end{array}\right], \mathbf{R}=\left[\begin{array}{ccccc}
3.05 & -2.23 & 1.66 & -0.10 & 0 \\
0 & -4.12 & 0.30 & -6.98 & 0 \\
0 & 0 & -0.02 & 0.11 & 0
\end{array}\right]
$$

The third column of $\mathrm{Q}$ is the direction cosine of the normal line of the plane. Numbers on the third row of $\mathrm{R}$ are the distances of each points to the fitted plane. Its equation can be obtained using point-slope form of plane:

$$
0.66(x-4)-0.72(y-4.6)+0.21(z-3)=0 \quad \Rightarrow \quad 0.66 x-0.72 y+0.21 z=0.042
$$

In solving this kind of problem, we can see that the 3-D concept and computer software are both extremely important. Hand calculation is useless and should be replaced.

\section{Conclusions}

Using these reform measures, the amount of theory is greatly reduced, useful theory has increased, the contents 
of theorem are significantly reduced; but the ability of solving practical problems has been considerably boosted. This book has set up a minimum theoretical limit to meet the needs of the engineering application objectively. Each content has its application background. Removing any part inevitable result in failure in solving some practical problems. The implementation of this book, plus MATLAB, need about 30 class hours, suitable for low class syllabus and continuing education. This book is also beneficial to the high hours curriculum. Because most of the existing textbooks are belong to the classical mathematical system. They cannot solve most of the application problems in this book. We can suggest this book as a reference in solving actual problems. All the textbooks can use these problems as the minimum engineering requirements, then use the spare time in their syllabus for higher requirements.

Postgraduate enrollment examination is an important factor affecting the reform of this course. Many exam problems were coming from classical linear algebra. Obviously the examination should be reformed to reduce useless theory and include some computer applications. Before the examination reform, we can leave these classical content into an follow-up elective course for senior students who want to take the examination.

\section{References}

[1] Chen, H.C. (2014) Applied Popular Linear Algebra with MATLAB. Xidian University Press.

[2] Tsien, H. (1998) The Retrospect and Prospect, Story of Old Jiaotong University. Jiangsu Literature and Art Publishing House.

[3] Carlson, D., et al. (1997) Resources for Teaching Linear Algebra. Mathematical Association of America Notes 42.

[4] You, H. and Zhu, G.J. (2012) Linear Algebra. High Education Press, Beijing, 97-114.

[5] Lay, D.C. (2006) Linear Algebra and Its Application. 3rd Edition, Pearson-Addison-Wesley, 162.

[6] Chen, H.C., Qu, S.L. and He, Y.J. (2010) Reduction of Complex Linear Systems Using Matrix Modeling. Proceedings of 29th Chinese Control Conference, Beijing, July.

[7] Strang, G. (2009) Introduction to Linear Algebra. 4th Edition, Wilsley-Cambridge Press, 244.

\section{Appendix: Define Determinant by Product of Diagonal Pivots [4] [7]}

While solving matrix equation $\mathbf{A X}=\mathbf{b}$ using Gauss elimination, the augmented matrix $\mathbf{C}=[\mathbf{A}$, $\mathbf{b}]$ is converted to a diagonal matrix $\mathrm{P}$ and a column vector $\mathrm{d}$ as follows:

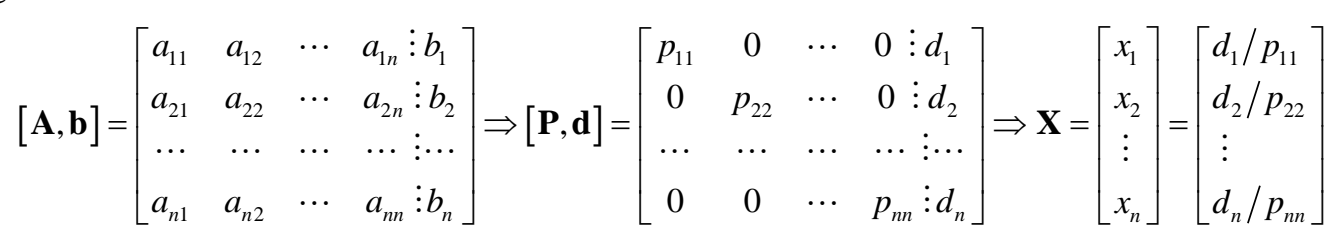

If all the pivots $\mathrm{p}_{11}, \mathrm{p}_{22}, \ldots, \mathrm{p}_{\mathrm{nn}}$ on the diagonal do not equal to zero, the solution of the equation is existed. This condition can be expressed in another way: Only if $|D|=\prod_{i=1}^{n}\left|p_{i i}\right| \neq 0$, the solution is existed. The product of diagonal pivots D is defined as determinant of matrix A. Here we discard its definition of sign to save paper. Actually we only want to know whether it equals to zero or not. Its exact value and sign are all unimportant. In most real world problems, people only want the solution, none requires determinant. So this definition is enough for most non math majors. In some cases like example-3, when some deeper concept is needed, classical theory won't help. The only way is to search subroutines in MATLAB. 